\title{
Serum neutrophil gelatinase-associated lipocalin for predicting acute pyelonephritis in infants with urinary tract infection
}

\author{
GRAŻYNA KRZEMIEŃ, MAŁGORZATA PAŃCZYK-TOMASZEWSKA, IWONA KOTUŁA, \\ URSZULA DEMKOW, AGNIESZKA SZMIGIELSKA \\ Medical University of Warsaw, Poland
}

\begin{abstract}
Introduction: Fever and elevated inflammatory markers have been used for diagnosis of acute pyelonephritis $(A P N)$ in infants with urinary tract infection (UTI).

The aim of the study was to compare the usefulness of serum neutrophil gelatinase-associated lipocalin ( $s N G A L)$ with inflammatory markers for predicting APN in infants with UTI.

Material and methods: The prospective study included 46 infants with a first episode of UTI, divided into two groups (APN 23, lower UTI 23), according to the DMSA scan results. The following laboratory tests were performed: $S N G A L, P C T, C R P, W B C$, and ESR.

Results: Significantly elevated levels of $S N G A L, P C T, C R P$, and ESR were observed in infants with APN compared to those with lower UTI. Higher sNGAL, CRP, and ESR values, presence of fever, and longer duration of fever before antibiotic treatment were associated with APN [odds ratio $(O R)$ 1.02, 1.27, 1.03, 13.46, 2.12, respectively]. Receiver operating characteristic (ROC) analysis showed better diagnostic profiles for $S N G A L, P C T$, and CRP than for ESR for predicting APN [area under the curve (AUC) 0.808, 0.819, 0.841, and 0.750, respectively]. The appropriate cut-off values of $S N G A L, P C T$ and CRP were $100.8 \mathrm{ng} / \mathrm{ml}, 0.15 \mathrm{ng} / \mathrm{ml}, 5.3 \mathrm{mg} / \mathrm{dl}$ (all sensitivity and specificity 82.6\%), and that of ESR was $40 \mathrm{~mm} / \mathrm{h}$ (sensitivity $78.3 \%$, specificity $60.9 \%$ ).

Conclusions: $S N G A L$ shows similar usefulness as PCT and CRP for predicting APN in infants with UTI, the diagnostic value of ESR is smaller, and WBC is not useful at all. The presence of fever and longer duration of fever are important predictors of APN in infants with UTI.
\end{abstract}

Key words: acute pyelonephritis, urinary tract infection, biomarkers, neutrophil gelatinaseassociated lipocalin, dimercaptosuccinic acid, scintigraphy, children.

(Centr Eur J Immunol 2019; 44 (1): 45-50)

\section{Introduction}

Urinary tract infection (UTI) is one of the most common bacterial infections in infants and young children, with an incidence of $2 \%$ during the first 2 years of life $[1,2]$. The prevalence of UTI is higher (5-24.2\%) among children aged $<2$ years presenting with fever [3-5]. UTI may be limited to the lower urinary tract or involve the renal parenchyma. Early diagnosis of acute pyelonephritis (APN) is difficult during infancy, because of nonspecific clinical symptoms of UTI at this age [6]. Delayed diagnosis and treatment of APN can cause long-term renal sequelae, such as renal scarring, hypertension, proteinuria, pregnancyrelated complications, and chronic kidney disease (CKD) $[7,8]$. In clinical practice, fever and acute-phase inflammatory markers such as elevation of white blood cells (WBC),
C-reactive protein (CRP), procalcitonin (PCT), and erythrocyte sedimentation rate (ESR) have been used to differentiate between APN and lower UTI $[9,10]$. However, these systemic inflammatory markers do not accurately localize the site of the UTI $[11,12]$.

99mTc-dimercaptosuccinic acid (DMSA) renal scintigraphy is the most sensitive standard diagnostic test for APN and renal scars in children. However, the routine use of the DMSA scan for localization of UTI is not recommended [3]. DMSA scanning has a lot of limitations such as high costs, time-consuming, injection, exposure to radiation, low accessibility, and difficulty of distinguishing between new renal parenchymal involvement and old scarring $[13,14]$. Renal power Doppler ultrasonography (US) may be helpful in diagnosis of APN, but it is not sensitive enough to give reliable information about renal

Correspondence: Agnieszka Szmigielska, MD, PhD, Medical University of Warsaw, Poland, e-mail: agnieszka.szmigielska@wum.edu.pl Submitted: 22.09.2018; Accepted: 17.12.2018 
parenchymal involvement $[7,15]$. Therefore, new, sensitive methods are needed for accurate diagnosis of APN in young children.

Neutrophil gelatinase-associated lipocalin (NGAL), a member of the lipocalin family, is an excellent biomarker for predicting and monitoring acute kidney injury (AKI) of diverse etiologies [16-19]. It is a promising marker for detection of subclinical renal damage due to vesicoureteral reflux (VUR) [20], renal scarring [21], and obstructive uropathy $[22,23]$. NGAL plays an important role in the innate immune response to the bacterial infection. It is released from activated neutrophils and other immune cells, and reduces bacterial growth in the early stage of infection [24, 25]. Recent reports in the literature demonstrated the utility of NGAL for predicting UTI [25-28] and APN [10, 29, 30] in children and adults. However, the published studies are small so far [2, 9, 27], only limited data are available for infants [1, 2, 5, 9, 31], and results are contentious [14, 32, 33]. The aim of this study was to compare the usefulness of serum NGAL (sNGAL) with systemic inflammatory markers for predicting APN in infants with UTI.

\section{Material and methods}

This prospective observational study was conducted in the Department of Pediatric and Nephrology over a period of 3 years. The study was approved by the local Bioethics Committee for Human Research and written informed consent was obtained from all parents. Forty-six infants (age range: 1-12 months) with the first episode of febrile or non-febrile UTI were enrolled in the study. Children with other infectious disease, AKI, CKD, obstructive nephropathy, and moderate or severe grade of vesicoureteral reflux (VUR) were excluded from the study. All children were investigated with a DMSA renal scan within 7 days after admission, to distinguish APN from lower UTI. APN was defined as focal, multifocal or diffusely decreased or absent cortical uptake, without cortical volume loss in which the renal cortical contour remains intact $[25,26]$. Children with normal scintigraphy were considered as having lower UTI. Patients were divided into two groups, APN and lower UTI, according to DMSA scan results. Urinalysis, urine culture, serum PCT, CRP, WBC, ESR and creatinine $(\mathrm{Cr})$ were performed on admission. Urine samples were obtained by bladder catheterization or clean mid-stream. Pyuria was defined as $>5$ WBC per high power field in the urine sediment, and significant bacteriuria as growth of a single pathogen $>100000 \mathrm{col}-$ ony-forming units per ml of urine [3]. The UTI diagnosis was made on the basis of the presence of both pyuria and significant bacteriuria [3]. Escherichia coli was isolated in $42(91 \%)$ infants, and other bacteria in $4(9 \%)$ infants (Klebsiella sp. in two children and Enterococcus sp. in two children). Fever was defined as a temperature of at least $38.0^{\circ} \mathrm{C}$. Normal values of evaluated indices in the local laboratory were as follows: $\mathrm{PCT}<0.05 \mathrm{ng} / \mathrm{ml}$, $\mathrm{CRP} \leq 1.0 \mathrm{mg} / \mathrm{dl}, \mathrm{ESR} \leq 12 \mathrm{~mm} / \mathrm{h}, \mathrm{Cr} \leq 0.4 \mathrm{mg} / \mathrm{dl}$. Leukocytosis was defined as a WBC count more than the normal value according to age. Serum samples for measurement of sNGAL were collected at the time of admission. They were immediately centrifuged and stored at $-80^{\circ} \mathrm{C}$ until further analysis. sNGAL levels were measured by an enzyme-linked immunosorbent assays (ELISA) kit (Human Lipocalin-2/NGAL; Cat no: RD 191102200R; Bio Vendor Laboratory Medicine, Inc. Brno, Czech Republic). The detection range for NGAL was $0.3-10 \mathrm{ng} / \mathrm{ml}$. US of the kidney and bladder was performed after admission. Hydronephrosis was classified according to the Society for Fetal Urology classification [34]. Voiding cystourethrography (VCUG) was performed 2-3 weeks after treatment of UTI in children with abnormal findings on US, or atypical UTI. The severity of VUR was classified according to the International Reflux Study Committee classification [35].

\section{Statistical analysis}

Statistical analysis was performed using the commercial Statistica package vs. 11.0 for Windows (StatSoft; Tulsa, OK). The distribution of continuous variables was checked for normality using the Lilliefors and Shapiro-Wilk tests. Data were presented as the mean \pm standard deviation (SD) or medians with corresponding $25^{\text {th }}$ and $75^{\text {th }}$ percentiles (IQR). Parametric Student's $t$-test or the non-parametric Mann-Whitney $U$ test was used to compare continuous variables between two groups. Categorical data were presented as numbers and percentiles. The chi-squared test was used to compare categorical variables between two groups. Correlations between variables were evaluated by linear regression analysis or Spearman's rank correlation. Univariate and multivariate logistic regression analysis was performed including the $95 \%$ confidence interval $(\mathrm{CI})$ to identify clinical variables associated with the presence of APN and to find an independent predictor of APN. Receiver operating characteristic (ROC) curve analysis was performed and the area under the curve (AUC) was calculated including the $95 \%$ CI to determine the best cut-off value, sensitivity and specificity for sNGAL and systemic inflammatory markers to predict APN. Statistical significance was set at $p<0.05$.

\section{Results}

We evaluated 46 infants (26 boys, 20 girls, mean age $5.39 \pm 3.15$ months) with the first episode of UTI. According to DMSA results, 23 (50\%) infants were included in the APN group, and $23(50 \%)$ in the lower UTI group. Demographic and laboratory data of children with APN and lower UTI are presented in Table 1. The children with APN 
were significantly older than those with lower UTI. Gender distribution did not differ between the groups. Fever was present significantly more often, and duration of fever before antibiotic treatment was significantly longer in the APN group compared to the lower UTI group. The median sNGAL level and values of PCT, CRP and ESR were significantly higher in children with APN compared to those with lower UTI. No significant differences in WBC count were found between children with APN and lower UTI. Four children with non-febrile UTI presented a positive DMSA scan. These children, despite the temperature below $38^{\circ} \mathrm{C}$, received anti-fever drugs at home. Therefore we could not document fever in this instance. Because of high inflammatory markers, and renal parenchymal involvement in the DMSA scan, we assigned these children to the APN group. Cr was normal in all children evaluated in the study. Congenital anomalies of the kidney and urinary tract (CAKUT) was diagnosed in $12(26 \%)$ children, including five with APN (three children with mild hydronephrosis, two children with VUR grade 2) and seven with lower UTI (three children with mild hydronephrosis, two children with VUR grade 2, and two children with duplex kidney).

Positive correlations were found between SNGAL, and PCT, CRP, WBC in the APN group ( $r=0.61, p<0.01$; $r=0.42, p<0.05 ; r=0.74, p<0.0001$, respectively); and between sNGAL, and age, fever duration, PCT, CRP, WBC, ESR in the total UTI group $(r=0.29, p<0.05 ; r=0.51$, $p<0.0001 ; r=0.84, p<0.0001 ; r=0.63, p<0.0001 ; r=0.71$, $p<0.0001 ; r=0.43, p<0.0001$, respectively). Univariate logistic regression analysis identified several clinical, and laboratory variables associated with APN, as shown in Table 2. This analysis showed that presence of fever, and longer duration of fever were important diagnostic factors of APN in infants. Multivariate analysis did not identify independent predictors of APN (data not shown). Area under the curve calculated with the ROC method, optimal cut-off value, sensitivity and specificity for SNGAL, and systemic inflammatory markers are presented in Table 3. ROC analysis demonstrated good diagnostic profiles (AUC, sensitivity and specificity) for SNGAL, PCT, and CRP. The diagnostic value of ESR was lower, and WBC was not useful for predicting APN.
Table 1. Demographic and laboratory data of children with acute pyelonephritis (APN) and lower urinary tract infection (UTI)

\begin{tabular}{|c|c|c|c|}
\hline \multirow[t]{2}{*}{ Variables } & \multicolumn{2}{|c|}{ Group } & \multirow[t]{2}{*}{$p$} \\
\hline & $\begin{array}{c}\text { APN } \\
n=23\end{array}$ & $\begin{array}{c}\text { Lower UTI } \\
n=23\end{array}$ & \\
\hline Age (months) & $6.39 \pm 3.70$ & $4.39 \pm 2.24$ & $<0.05$ \\
\hline Male, $n(\%)$ & $12(52.2)$ & $14(60.8)$ & ns \\
\hline $\begin{array}{l}\text { Fever } \geq 38^{\circ} \mathrm{C} \\
\text { present } n(\%)\end{array}$ & $19(82.6)$ & $6(26.1)$ & $<0.001$ \\
\hline $\begin{array}{l}\text { Fever duration } \\
\text { (days) }\end{array}$ & $3.00(1.0-4.0)$ & $0.00(0.0-1.0)$ & $<0.001$ \\
\hline sNGAL (ng/ml) & $130.5(100.8-205.8)$ & $57.6(39.0-72.3)$ & $<0.001$ \\
\hline PCT (ng/ml) & $0.47(0.19-2.05)$ & $0.08(0.05-0.13)$ & $<0.001$ \\
\hline $\mathrm{CRP}(\mathrm{mg} / \mathrm{dl})$ & $7.00(5.3-16.4)$ & $1.8(0.5-3.8)$ & $<0.0001$ \\
\hline $\mathrm{WBC}\left(\mathrm{mm}^{3}\right)$ & $17.62 \pm 7.43$ & $14.70 \pm 4.37$ & ns \\
\hline $\operatorname{ESR}(\mathrm{mm} / \mathrm{h})$ & $60.0(40.0-105.0)$ & $35.0(14.0-50.0)$ & $<0.001$ \\
\hline
\end{tabular}

Table 2. Univariate logistic regression analysis to identify risk factors associated with the presence of acute pyelonephritis (APN)

\begin{tabular}{lcc}
\hline Variable & OR $(\mathbf{9 5 \%}$ CI $)$ & $\boldsymbol{p}$ \\
\hline Age (months) & $1.25(1.01-1.53)$ & $<0.05$ \\
\hline Fever present & $13.46(3.24-55.92)$ & $<0.0001$ \\
\hline Fever duration (days) & $2.12(1.35-3.31)$ & $<0.01$ \\
\hline sNGAL & $1.02(1.00-1.03)$ & $<0.01$ \\
\hline PCT & $1.24(0.89-1.73)$ & $\mathrm{ns}$ \\
\hline CRP & $1.27(1.08-1.49)$ & $<0.01$ \\
\hline WBC & $1.09(0.98-1.21)$ & $\mathrm{ns}$ \\
\hline ESR & $1.03(1.01-1.06)$ & $<0.01$ \\
\hline
\end{tabular}

OR-odds ratio, CI-confidence interval, sNGAL-serum neutrophil gelatinaseassociated lipocalin; $P C T$ - procalcitonin; $C R P-C$-reactive protein, $W B C-$ white blood cell count; ESR - erythrocyte sedimentation rate

Table 3. Area under the curve (AUC), optimal cut-off value, sensitivity and specificity of serum neutrophil gelatinaseassociated lipocalin (sNGAL), and systemic inflammatory markers to predict acute pyelonephritis (APN)

\begin{tabular}{lcccccc}
\hline Variable & AUC $(\mathbf{9 5 \%}$ CI) & $\boldsymbol{z}$ & $\boldsymbol{p}$ & Cut-off & Sensitivity (\%) & Specificity (\%) \\
\hline sNGAL $(\mathrm{ng} / \mathrm{ml})$ & $0.808(0.673-0.943)$ & 4.47 & $<0.0001$ & 100.80 & 82.60 & 82.60 \\
\hline PCT $(\mathrm{ng} / \mathrm{ml})$ & $0.819(0.688-0.951)$ & 4.76 & $<0.0001$ & 0.15 & 82.60 & 82.60 \\
\hline CRP $(\mathrm{mg} / \mathrm{dl})$ & $0.841(0.724-0.958)$ & 5.71 & $<0.0001$ & 5.30 & 82.60 & 82.60 \\
\hline WBC $\left(\mathrm{mm}^{3}\right)$ & $0.617(0.450-0.784)$ & 1.38 & $\mathrm{~ns}$ & - & - & - \\
\hline ESR $(\mathrm{mm} / \mathrm{h})$ & $0.750(0.609-0.890)$ & 3.48 & $<0.001$ & 40.00 & 78.30 & 60.90 \\
\hline
\end{tabular}

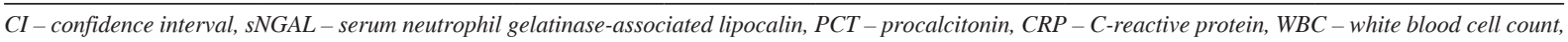
ESR - erythrocyte sedimentation rate 


\section{Discussion}

The early diagnosis and treatment of APN in infants are important to prevent or reduce long-term renal sequelae [7]. The DMSA scan has been considered as a gold standard for detecting APN, but it has well-known limitations $[3,13]$. Therefore, it is important to find more practical methods to identify APN in infants and young children. At the present, several biomarkers have been investigated for APN in children [1, 36, 37].

In 2009 Ichino et al., in an experimental rat UTI model, demonstrated a marked increase of urine NGAL (uNGAL) at the early stage of infection. The authors suggested the potential utility of UNGAL as a marker of APN in humans [38]. Since that time, several clinical studies have been conducted for assessing the diagnostic value of UNGAL for detecting APN, but the results are not clear [14, 29, 33, 39]. In children, measurement of urine biomarkers is preferred over blood tests because of the less painful and non-invasive procedure [25]. Therefore, only limited data are available about the utility of blood NGAL for early prediction of UTI and APN in children [2, 9, 10, 30, 32, 40]. Kim et al. found significantly higher levels of sNGAL in children with pyuria-positive UTI compared to those with pyuria-negative UTI [32]. Petrovic et al. reported a significantly higher sNGAL level in children with UTI at the time of admission to the hospital than at the time of hospital discharge [40]. Four pediatric studies have evaluated the utility of plasma NGAL (pNGAL) for predicting APN [2, 9, 10, 30]. Kim et al. evaluated 138 children, and Sim et al. 123 children with febrile UTI. The authors demonstrated good diagnostic profiles of pNGAL for predicting APN (AUC 0.893 and 0.864 , sensitivity $86 \%$ and $89.1 \%$, specificity $85 \%$ and $71 \%$, respectively) [10, 30]. Yun et al. performed a study in 64 infants, and Seo et al. in 47 infants with febrile UTI, and reported worse diagnostic profiles for pNGAL according to the reports outlined above (AUC 0.745 and 0.748 , sensitivity $72.1 \%$ and $75 \%$, specificity $71.4 \%$ and $78.3 \%$, respectively) $[2,9]$. Because in our previous study sNGAL was an excellent marker for early diagnosis of febrile UTI in infants [31], in our present study we compared the usefulness of sNGAL with systemic inflammatory markers for predicting APN in infants with UTI. However, some researchers prefer to use plasma samples, as NGAL may be released from neutrophils during the preparation of serum [2]. We revealed better AUC (0.808), sensitivity (82.6\%), and specificity (82.6\%) of sNGAL for predicting APN in infants than reported by Yun et al. and Seo et al. [2, 9]. The suggested cut-off value of sNGAL (100.8 ng/ml) for APN in our study could not be compared with the findings of Yun et al. $(267 \mu \mathrm{g} / \mathrm{l})$ and Seo et al. $(61.0 \mathrm{ng} / \mathrm{ml})$, because of the difference in measurement method between SNGAL and pNGAL [2, 9].

Numerous studies have evaluated the usefulness of inflammatory markers for predicting APN, and demonstrated that commonly used laboratory parameters such as
WBC, CRP and ESR have low sensitivity and specificity for accurately predicting acute renal parenchymal involvement. At present, these tests are not recommended for routine use to differentiate APN from lower UTI in children $[11,12,14]$. A previous meta-analysis showed that PCT was unable to predict APN in children [12]. In a recent meta-analysis Zhang et al. reported that a PCT cut-off value of $1.0 \mathrm{ng} / \mathrm{ml}$ is preferable to a value of $0.5 \mathrm{ng} / \mathrm{ml}$ for predicting APN in young children. The authors concluded that further multicenter studies are needed to validate this cut-off value of PCT for predicting APN [41]. Only a few pediatric studies have compared the usefulness of pNGAL for prediction of APN to the conventional inflammatory markers. Kim et al. revealed high AUC values for pNGAL (0.893), CRP (0.879), and PCT (0.855), and lower for WBC (0.654). In this study AUC values did not differ significantly, except between pNGAL and WBC [10]. Yun et al. Seo et al. Sim et al. also reported high AUC for pNGAL (0.745 to 0.864), followed by CRP (0.691 to 0.842$)$ and WBC (0.642 to 0.698) $[2,9,30]$. Yun et al., Kim et al., Seo et al. demonstrated that pNGAL was an independent predictor of APN [2, 10, 30]. Based on the results of our study, we found similar AUC for sNGAL (0.808), CRP (0.841), and PCT (0.819). sNGAL had the same sensitivity and specificity as CRP and PCT for predicting APN in infants. The diagnostic value of ESR was lower (0.750), and WBC was not useful at all for predicting APN. Multivariate analysis did not identify independent predictors of APN. We noted positive correlations between sNGAL and PCT, CRP, WBC in the APN group; and between sNGAL and PCT, CRP, WBC, ESR in the total UTI group. In contrast, Sim et al. demonstrated no correlation of pNGAL with CRP, WBC, and ESR in the APN group and the total UTI group [30].

Fever is commonly used for predicting APN in children. However, the prevalence of an abnormal DMSA scan in infants with febrile UTI varies from $16.3 \%$ to $67 \%$ [2, $9,13]$. In our study $50 \%$ of children with UTI presented renal parenchymal involvement. Similar to reports of Bańuelos-Andrio et al. and Pathirana et al., we evaluated infants with febrile and non-febrile UTI $[11,42]$. Four children with non-febrile UTI presented a positive DMSA scan. These children, despite the temperature being below $38^{\circ} \mathrm{C}$, received anti-fever drugs at home. Sometimes fever lasts only a few hours in children with UTI [13, 42]. So we could not exclude higher temperature in these infants before admission. The temperature during hospitalization was also $<38^{\circ} \mathrm{C}$. Because of high inflammatory markers and renal parenchymal involvement in the DMSA scan we assigned these infants to the APN group. Some authors demonstrated that renal parenchymal involvement in UTI is related to delayed treatment and longer duration of fever before antibiotic treatment $[8,13,30,43]$. In our study fever $\geq 38^{\circ} \mathrm{C}$ was present significantly more often and duration of fever before antibiotic treatment was significantly longer in the group with APN compared to the lower UTI 
group. Univariate analysis showed that the presence of fever and the duration of fever were important diagnostic factors of APN in infants. In contrast, Yun et al. and Seo et al. noted no difference in duration of fever before treatment between APN and lower UTI groups $[2,9]$.

The main source of blood NGAL in UTI is activated peripheral neutrophils in the circulation and in the infected kidney $[16,24]$. Children with APN may develop prerenal type of AKI as a result of dehydration and hypoperfusion. Severe forms of APN can lead to acute, mostly tubule-interstitial kidney injury, even in the presence of normal $\mathrm{Cr}$ levels $[10,30,40]$. In patients with the severe APN and concomitant AKI, NGAL is massively upregulated in the distal part of the nephron (thick ascending limb of Henle's loop, distal tubule, and collecting duct) into blood and urine [16]. In pediatric studies, acute elevation and rapid decline of blood NGAL showed that the source of NGAL elevation was neutrophils rather than injured cells in the distal tubule [6, 37, 40]. uNGAL derives mainly from activated neutrophils present in urine, less from injured epithelium [32, 44]. Foster et al. suggested that pNGAL is a good marker of systemic inflammation associated with APN and renal damage, while UNGAL is effective for detecting infection in the genitourinary system. Elevated pNGAL and uNGAL levels suggest the presence of systemic inflammation associated with APN, and low pNGAL and elevated UNGAL levels indicate lower UTI, which has not led to systemic inflammation [22].

There were some limitations of our study. We evaluated only serum NGAL concentration, but not urine NGAL concentration. This was a single-center study, with a small cohort of children, and a difference in children's age between the groups. It is difficult to have closely agematched groups in observational studies. In the present study, infants with APN were significantly older than those with lower UTI, but the difference were small, around two months. Therefore, it seems unlikely that higher levels of sNGAL in the APN group than those in the lower UTI group were associated with the age difference. However, a few authors have reported a higher probability of APN occurring in older children than in younger children $[1,42$, 45]. Unfortunately, reliable reference intervals for pNGAL and sNGAL are lacking for healthy infants.

\section{Conclusions}

In the present study we demonstrated that SNGAL shows similar utility as PCT and CRP for predicting APN in infants with UTI. The diagnostic value of ESR for predicting APN in infants is smaller, and WBC is not useful at all. The presence of fever and longer duration of fever are important predictors of APN in infants with UTI.

The authors declare no conflict of interest.

\section{References}

1. Valdimarsson S, Jodal U, Barregård L, et al. (2017): Urine neutrophil gelatinase-associated lipocalin and other biomarkers in infants with urinary tract infection and in febrile controls. Pediatr Nephrol 32: 2079-2087.

2. Yun BA, Yang EM, Kim CJ (2018): Plasma neutrophil gelatinase-associated lipocalin as a predictor of renal parenchymal involvement in infants with febrile urinary tract infection: a preliminary study. Ann Lab Med 38: 425-430.

3. Subcommittee on Urinary Tract Infection, Steering Committee on Quality Improvement and Management (2011): urinary tract infection: clinical practice guideline for the diagnosis and management of the initial UTI in febrile infants and children 2 to 24 months. Pediatrics 128: 595-610.

4. Lubell TR, Barasch JM, Xu K, et al. (2017): Urinary neutrophil gelatinase-associated lipocalin for the diagnosis of urinary tract infections. Pediatrics 140: e20171090.

5. Jung N, Byun HJ, Park JH, et al. (2018): Diagnostic accuracy of urinary biomarkers in infants younger than 3 months with urinary tract infection. Korean J Pediatr 61: 24-29.

6. Lee H-E, Kim DK, Kang HK, et al. (2015): The diagnosis of febrile urinary tract infection in children may be facilitated by urinary biomarkers. Pediatr Nephrol 30: 123-130.

7. Marceau-Grimard M, Marion A, Côté C, et al. (2017): Dimercaptosuccinic acid scintygrafphy vs. ultrasound for renal parenchymal defects in children. Can Urol Assoc 11: 260264.

8. Karavanaki KA, Soldatou A, Koufadaki AM, et al. (2017): Delayed treatment of the first febrile urinary tract infection in early childhood increased the risk of renal scarring. Acta Pediatr 106: 149-154.

9. Seo WH, Nam SW, Lee EH, et al. (2014): A rapid plasma neutrophil gelatinase-associated lipocalin assay for diagnosis of acute pyelonephritis in infants with acute febrile urinary tract infections: a preliminary study. Eur J Pediatr 173: 229-232.

10. Kim BK, Yim HE, Yoo KH (2017): Plasma neutrophil gelatinase-associated lipocalin: a marker of acute pyelonephritis in children. Pediatr Nephrol 32: 477-484.

11. Bańuelos-Andrio L, Espino-Hernández M, Ruperez-Lucas M, et al. (2017): Usefulness of analytical parameters in the management of pediatric patients with suspition of acute pyelonephritis. Is procalcitonin reliable? Rev Esp Med Nucl Imagen Mol 36: 2-6.

12. Shaikh N, Borrell JL, Evron J, et al. (2015): Procalcitonin, C-reactive protein, and erythrocyte sedimentation rate for the diagnosis of acute pyelonephritis in children. Cochrane Database Syst Rev doi: 10.1002/14651858.CD009185.pub2

13. Printza N, Farmaki E, Piretzi K, et al. (2012): Acute phase 99mTc-dimercaptosuccinic acid scan in infants with first episode of febrile urinary tract infection. World J Pediatr 8: 52-56.

14. Ghasemi K, Esteghamati M, Borzoo S, et al. (2016): Predictive accuracy of urinary neutrophil gelatinase associated lipocalin (NGAL) for renal parenchymal involvement in children with acute pyelonephritis. Electron Physician 8: 1911-1917.

15. Ayazi P, Mahyar A, Noroozian E, et al. (2015): Comparison of renal ultrasonography and dimercaptosuccinic acid renal scintigraphy in febrile urinary tract infection. Infez Med 4: 323-329.

16. Singer E, Markó L, Paragas N, et al. (2013): Neutrophil gelatinase-associated lipocalin: pathophysiology and clinical applications. Acta Physiol (Oxf) 207: 663-672.

17. Kisiel A, Roszkowska-Blaim M, Pańczyk-Tomaszewska M, et al. (2017): Effect of perinatal risk factors on neutrophil 
gelatinase-associated lipocalin (NGAL) level in umbilical and peripheral blood in neonates. Cent Eur J Immunol 3: 274-280.

18. Filho LT, Grande AJ, Colonetti T, et al. (2017): Accuracy of neutrophil gelatinase-associated lipocalin for acute kidney injury diagnosis in children: systematic review and meta-analysis. Pediatr Nephrol 32: 1979-1988.

19. Lichosik M, Jung K, Jobs K, et al. (2015): Interleukin-18 and neutrophil gelatinase-associated lipocalin in assessment of the risk of contrast-induced nephropathy in children. Cent Eur J Immunol 40: 447-453.

20. Parmaksiz G, Noyan A, Dursun H, et al. (2016): Role of new biomarkers for predicting renal scarring in vesicoureteral reflux: NGAL, KIM-1, and L-FABP. Pediatr Nephrol 31: $97-$ 103.

21. Rafiei A, Mohammadjafari H, Bazi S, et al. (2015): Urinary neutrophil gelatinase-associated lipocalin (NGAL) might be an independent marker for anticipating scar formation in children with acute pyelonephritis. J Renal Inj Prev 4: 39-44.

22. Forster CS, Davarajan P (2017): Neutrophil gelatinase-associated lipocalin: utility in urologic conditions. Pediatr Nephrol 32: $377-381$

23. Gupta S, Jackson AR, DaJusta DG, et al. (2018): Urinary antimicrobial peptides: Potential novel biomarkers of obstructive uropathy. J Pediatr Urol 14: 238.e1-238.e6.

24. Nasioudis D, Witkin SS (2015): Neutrophil gelatinase-associated lipocalin and innate immune responses to bacterial infections. Med Microbiol Immunol 204: 471-479.

25. Ylmaz A, Sevketoglu E, Gedikbasi A, et al. (2009): Early prediction of urinary tract infection with urinary neutrophil gelatinase associated lipocalin. Pediatr Nephrol 24: 23872392.

26. Hatipoglu S, Sevketoglu E, Gedikbasi A, et al. (2011): Urinary MMP-9/NGAL complex in children with acute cystitis. Pediatr Nephrol 26: 1263-1268.

27. Price JR, Guran L, Lim JY, et al. (2017): Neutrophil gelatinase-associated lipocalin biomarker and urinary tract infections: a diagnostic case-control study (NUTI Study). Female Pelvic Med Reconstr Surg 23: 101-107.

28. Forster CS, Jackson E, Ma Q, et al. (2018): Predictive ability of NGAL in identifying urinary tract infection in children with neurogenic bladders. Pediatr Nephrol 33: 1365-1374.

29. Arambašic J, Mandić S, Debeliak Ž, et al. (2016): Differentiation of acute pyelonephritis from other febrile states in children using urinary neutrophil gelatinase-associated lipocalin (uNGAL). Clin Chem Lab Med 54: 55-61.

30. Sim JH, Yim HE, Choi BM, et al. (2015): Plasma neutrophil gelatinase-associated lipocalin predicts acute pyelonephritis in children with urinary tract infections. Pediatr Res 78: 48-55.

31. Krzemień G, Pańczyk-Tomaszewska, Adamczuk D, et al. (2018): Neutrophil gelatinase-associated lipocalin: a biomarker for early diagnosis of urinary tract infections in infants. Advs Exp Med Biol 38: 71-80.

32. Kim BH, Yu N, Kim HR, et al. (2014): Evaluation of the optimal neutrophil gelatinase-associated lipocalin value as a screening biomarker for urinary tract infections in children. Ann Lab Med 34: 354-359.

33. Urbschat A, Obermüller N, Paulus P, et al. (2014): Upper and lower urinary tract infections can be detected early but not be discriminated by urinary NGAL in adults. Int Urol Nephrol 46: 2243-2249.

34. Fernbach S, Maizels M, Conway J (1993): Ultrasound grading of hydronephrosis: introduction to the system used by the Society for Fetal Urology. Pediatr Radiol 23: 478-480.
35. Lebowitz RL, Olbing H, Parkkulainen KV, et al. (1985): International reflux study in children: international system of radiographic grading of vesicoureteral reflux. Pediatr Radiol 15: 105-109.

36. Krzemień G, Szmigielska A, Turczyn A, et al. (2016): Urine interleukin-6, interleukin-8 and transforming growth factor $\beta 1$ in infants with urinary tract infection and asymptomatic bacteriuria. Centr Eur J Immunol 41: 260-267.

37. Yim HE, Yim H, Bae ES, et al. (2014): Predictive value of urinary and serum biomarkers in young children with febrile urinary tract infections. Pediatr Nephrol 29: 2181-2189.

38. Ichino M, Kuroyanagi Y, Kusaka M, et al. (2009): Increased urinary neutrophil gelatinase associated lipocalin levels in a rat model of upper urinary tract infection. J Urol 181: 23262331.

39. Nickavar A, Safaeian B, Valavi E, et al. (2016): Validity of neutrophil gelatinase associated lipocaline as a biomarker for diagnosis of children with acute pyelonephritis. Pediatr Urol 13: 2860-2863.

40. Petrovic S, Bogavac-Stanojevic N, Peco-Antic A, et al. (2013): Clinical application neutrophil gelatinase-associated lipocalin and kidney injury molecule- 1 as indicators of inflammation persistence and acute kidney injury in children with urinary tract infection. Biomed Res Int 2013: 947157.

41. Zhang H, Yang J, Lin L, et al. (2016): Diagnostic value of serum procalcitonin for acute pyelonephritis in infants and children with urinary tract infections: an updated meta-analysis. World J Urol 34: 431-441.

42. Pathirana KG, Fernando J, Samarasinghe N, et al. (2016): Usefulness of $99 \mathrm{mTcDimercaptosuccinic}$ acid scan safter the first episode of urinary tract infection in children admitted to the Lady Ridgeway Hospital for Children, Sri Lanka. Ceylon Med J 61: 80-82.

43. Gilani KA, Esfeh JM, Gholamrezanezhad A, et al. (2010): Predictors of abnormal renal cortical scintigraphy in children with first urinary tract infection: the importance of time factor. Int Urol Nephrol 42: 1041-1047.

44. Decavele AS, Dhondt L, De Buyzere ML, et al. (2011): Increased urinary neutrophil gelatinase associated lipocalin in urinary tract infection and leukocyturia. Clin Chem Lab Med 49: 999-1003.

45. Pecile P, Miorin E, Romanello C, et al. (2009): Age-related renal parenchymal lesions in children with first febrile urinary tract infections. Pediatrics 124: 23-29. 